\title{
VIBRATION CONTROL OF STRUCTURES WITH UNCERTAINTIES DUE TO COUPLED SUBSYSTEMS
}

\author{
J. Rodellar*, V. Mañosa ${ }^{\dagger}$, E. Reithmeier**, G. Ehret ${ }^{* *}$ \\ * Departamento de Matemàtica Aplicada III, Universitat Politècnica de Catalunya, \\ Jordi Girona 1-3, 08034 Barcelona, Spain \\ fax: +3493401 1825 \\ e-mail: jose.rodellar@upc.es \\ † Departamento de Matemàtica Aplicada III, Universitat Politècnica de Catalunya, \\ Colom 1, 08222 Terrassa, Spain \\ fax: $\quad+34937398225$ \\ e-mail: victor.manosa@upc.es \\ ** Institut für Mess und Regelungstechnik, Universität Hannover, \\ Nienburger Str., 17D-30167 Hannover, Germany \\ fax: $\quad+490 \quad 511762 \quad 3234$ \\ e-mail: reithmeier@imr.uni-hannover.de, \\ ehreteimr.uni-hannover.de
}

Keywords: Active vibration control, Robust control, Lyapunov stability theory.

\begin{abstract}
Vibrations in dynamical structures, as those encountered for instance in mechanical, aerospace or civil engineering, are often caused by internal or external excitations. One way to attenuate vibrations that can be undesirable for safety or other reasons is by the use of active control systems. This means to use feedback controllers driving actuators at appropriate locations between the structure and its environment. This work considers a specific kind of uncertain excitation due to a temporary coupling of the structure with a second dynamical system which cannot be influenced by actuators controlling the main structure. A linear control scheme is proposed, which is implemented with the feedback of the structure's state only and ensures the existence of a ball of ultimate boundedness. The effectiveness of the control scheme is illustrated by numerical experiments on a model of a bridge platform with crossing vehicles as unknown coupling excitations.
\end{abstract}

\section{Introduction}

Active control has grown in the last years as a mean to attenuate vibrations of dynamical flexible structures subjected to internal or external excitations. Examples are encountered in areas such as mechanical, aerospace or civil engineering. Active control means to use feedback controllers driving actuators at appropriate locations between the structure and its environment. In the last years different approaches have been taken from control theory to develop active controllers. Within this context, robust control methods have been used to account for uncertainties in the structural models and the lack of knowledge of the excitations, which in most of the cases are even unpredictable. One of the robust control approaches for this purpose has been based on the constructive use of Lyapunov stability techniques [1-3]. Within this approach, semiactive structural control systems have been developed for instance in [4-5]. In this kind of systems, the feedback is designed to modify in real time parameters such as damping or stiffness to increase the resistance of the structures as compared with the purely passive schemes. Purely active controllers, in which the feedback is used to feed actuators to implement desired forces to the structures, have been proposed for example in [6-7].

Following this direction, this paper deals with a class of structures excited by the coupling with another dynamical system during a certain time. An active linear controller is proposed which uses feedback information from the controlled structure only. It is shown that the response of the structure and the exciting system stay bounded within a neighborhood of the origin. The class of systems is illustrated by considering a model of a bridge platform with an unknown moving vehicle as coupled exciting system. Numerical results are given to show the effectiveness of the approach in getting a practical reduction in the vibrations of the platform.

\section{Control scheme}

The control objective is to attenuate the vibrations of a system described by the state space variables $\mathbf{x} \in \mathbb{R}^{n_{c}}$. The excitation applied to this system is due to a second system, described by the state space variables $\mathbf{y} \in \mathbb{R}^{n_{T}}$, to which it is coupled. This coupling is uncertain. In this sense, the following model is considered:

Main System: $\quad \dot{\mathbf{x}}=\mathbf{A}_{\mathbf{c}} \mathbf{x}+\mathbf{B}_{\mathbf{c}} \mathbf{u}+\mathbf{g}_{\mathbf{c}}(\mathbf{x}, \mathbf{w}, t)$, Exciting System: $\quad \dot{\mathbf{y}}=\mathbf{A}_{\mathbf{r}} \mathbf{y}+\mathbf{g}_{\mathbf{r}}(\mathbf{y}, \mathbf{w}, t)$.

$\mathbf{u} \in \mathbb{R}^{m}$ is the control, which acts only on the main system. $\mathbf{A}_{\mathbf{c}}, \mathbf{B}_{\mathbf{c}}$ and $\mathbf{A}_{\mathbf{r}}$ are known constant matrices of appropriate di- 
mensions. The coupling between the systems goes through the uncertain functions $\mathbf{g}_{\mathbf{c}}$ and $\mathbf{g}_{\mathbf{r}}$, and is due to some interchange action $\mathbf{w} \in \mathbb{R}^{\ell}$, which can be described by some mathematical relationship such as $\mathbf{w}=\mathbf{f}(\mathbf{x}, \mathbf{y}, t)$. In the case that there is no coupling, $\mathbf{w}=0$.

The following assumptions complete the model of the system.

Assumption 1: $\mathbf{A}_{\mathbf{c}}$ and $\mathbf{A}_{\mathbf{r}}$ are stable matrices.

Assumption 2: There exists an uncertain function $\mathbf{e}: \mathbb{R}^{n_{c}} \times$ $\mathbb{R}^{n_{r}} \times \mathbb{R} \longrightarrow \mathbb{R}^{n_{r}}$ such that $\mathbf{e}(\cdot, \cdot, t)$ is continuous and $\mathbf{e}(\mathbf{x}, \mathbf{y}, \cdot)$ is continuous for all $t$ except for a set $\left\{t_{1}, t_{2}, \ldots, t_{n}\right\}$, and there exist known non-negative constants $\alpha_{c}^{c}, \alpha_{c}^{r}, \delta_{c}, \mu_{r}^{c}$, and $\nu_{r}$ such that

(2.1) $\mathbf{g}_{\mathbf{c}}(\mathbf{x}, \mathbf{f}(\mathbf{x}, \mathbf{y}, t), t)=\mathbf{B}_{\mathbf{c}} \mathbf{e}(\mathbf{x}, \mathbf{y}, t)$,

(2.2) $\|\mathbf{e}(\mathbf{x}, \mathbf{y}, t)\| \leq \alpha_{c}^{c}\|\mathbf{x}\|+\alpha_{c}^{r}\|\mathbf{y}\|+\delta_{c}$,

(2.3) $\left\|\mathbf{g}_{\mathbf{r}}(\mathbf{x}, \mathbf{f}(\mathbf{x}, \mathbf{y}, t), t)\right\| \leq \mu_{r}^{c}|| \mathbf{x}\left\|+\mu_{r}^{r}|| \mathbf{y}\right\|+\nu_{r}$,

hold for all $\mathbf{x}, \mathbf{y}$ and $t$.

Consider the linear feedback control

$$
\mathbf{u}(t)=-\gamma \mathbf{B}_{\mathbf{c}}{ }^{T} \mathbf{K}_{\mathbf{c}} \mathbf{x}(t),
$$

where $\mathbf{K}_{\mathbf{c}}$ is the solution of the algebraic Lyapunov equation

$$
\mathbf{K}_{\mathbf{c}} \mathbf{A}_{\mathbf{c}}+\mathbf{A}_{\mathbf{c}}{ }^{T} \mathbf{K}_{\mathbf{c}}+\mathbf{Q}_{\mathbf{c}}=\mathbf{0}
$$

for a given matrix $\mathbf{Q}_{\mathbf{c}} \in \mathbb{R}^{n_{c}, n_{c}}$, positive definite. And where $\gamma>0$ is a gain parameter. Notice that the matrix $\mathbf{B}_{\mathbf{c}}{ }^{T} \mathbf{K}_{\mathbf{c}}$ of this control uses only information on the known part $\left(\mathbf{A}_{\mathbf{c}}, \mathbf{B}_{\mathbf{c}}\right)$ of the uncoupled main system, while the scalar gain $\gamma$ will be chosen through a stability analysis which involves the known part $\left(\mathbf{A}_{\mathbf{r}}\right)$ of the exciting system and the bounds on the uncertainties in Assumption 2.

The stability analysis will ensure that the control defined in (2), with an appropriate selection of $\gamma$, guarantees that there exists a parameter value $\rho>0$ such that any admissible trajectory $\xi(t)=(\mathbf{x}(t), \mathbf{y}(t))$ enters $\mathrm{B}_{\rho}=\left\{\xi \in \mathbb{R}^{n_{c}} \times \mathbb{R}^{n_{r}}:|\xi| \leq \rho\right\}$ at a time $T$, and that there exists a compact set $\mathcal{K}$, which is a global uniform attractor of (1).

\section{Stability analysis}

Let us define the following Lyapunov function candidate for system (1):

$V(\mathbf{x}, \mathbf{y}):=<\mathbf{x}, \mathbf{K}_{\mathbf{c}} \mathbf{x}>+<\mathbf{y}, \mathbf{K}_{\mathbf{r}} \mathbf{y}>=\left(\mathbf{x}^{T}, \mathbf{y}^{T}\right) \mathbf{K}\left(\begin{array}{l}\mathbf{x} \\ \mathbf{y}\end{array}\right)$

where $\mathbf{K}_{\mathbf{r}}$ is the solution of the algebraic Lyapunov equation

$$
\mathbf{K}_{\mathbf{r}} \mathbf{A}_{\mathbf{r}}+\mathbf{A}_{\mathbf{r}}^{T} \mathbf{K}_{\mathbf{r}}+\mathbf{Q}_{\mathbf{r}}=\mathbf{0}
$$

for a chosen matrix $\mathbf{Q}_{\mathbf{r}} \in \mathbb{R}^{n_{r}, n_{r}}$, positive definite, and $\mathbf{K}=$ $\operatorname{diag}\left(\mathbf{K}_{\mathbf{c}}, \mathbf{K}_{\mathbf{r}}\right)$. Thus

$$
\begin{aligned}
\frac{d}{d t} V(\mathbf{x}, \mathbf{y}) \leq & 2<\mathbf{x}, \mathbf{A}_{\mathbf{c}} \mathbf{x}>+2<\mathbf{x}, \mathbf{g}_{\mathbf{c}}(\mathbf{x}, \mathbf{w}, t)>+ \\
& 2<\mathbf{y}, \mathbf{A}_{\mathbf{r}} \mathbf{y}>+2<\mathbf{y}, \mathbf{g}_{\mathbf{r}}(\mathbf{x}, \mathbf{w}, t)>
\end{aligned}
$$

Using Assumption 2 and equations (3) and (4), we have:

$$
\begin{gathered}
\frac{d}{d t} V(\mathbf{x}, \mathbf{y}) \leq-<\mathbf{x}, \mathbf{Q}_{\mathbf{c}} \mathbf{x}>-2 \gamma\left\|\mathbf{B}_{\mathbf{c}}{ }^{T} \mathbf{K}_{\mathbf{c}} \mathbf{x}\right\|^{2}+ \\
2 \alpha_{c}^{c}\|\mathbf{x}\|\left\|\mathbf{B}_{\mathbf{c}}{ }^{T} \mathbf{K}_{\mathbf{c}} \mathbf{x}\right\|+2 \alpha_{c}^{r}\|\mathbf{y}\|\left\|\mathbf{B}_{\mathbf{c}}{ }^{T} \mathbf{K}_{\mathbf{c}} \mathbf{x}\right\|+ \\
+2 \delta_{c} \mid \mathbf{B}_{\mathbf{c}}{ }^{T} \mathbf{K}_{\mathbf{c}} \mathbf{x}\left\|-<\mathbf{y}, \mathbf{Q}_{\mathbf{r}} \mathbf{y}>+2 \mu_{r}^{c}\right\| \mathbf{K}_{\mathbf{r}}\|\| \mathbf{x}\|\| \mathbf{y} \|+ \\
+2 \mu_{r}^{r}\left\|\mathbf{K}_{\mathbf{r}}\right\|\|\mathbf{y}\|^{2}+2 \nu_{r}\left\|\mathbf{K}_{\mathbf{r}}\right\|\|\mathbf{y}\| .
\end{gathered}
$$

Using the fact that $-<\mathbf{x}, \mathbf{Q}_{\mathbf{c}} \mathbf{x}>\leq-\lambda_{\min }\left(\mathbf{Q}_{\mathbf{c}}\right)\|\mathbf{x}\|^{2}$ and also that $-<\mathbf{y}, \mathbf{Q}_{\mathbf{r}} \mathbf{y}>\leq-\lambda_{\min }\left(\mathbf{Q}_{\mathbf{r}}\right)\|\mathbf{y}\|^{2}$ (where $\lambda_{\text {min }}$ denotes the minimum eigenvalue), and setting

$$
\begin{aligned}
& \mathbf{z}:=\left(\|\mathbf{x}\|,\|\mathbf{y}\|,\left\|\mathbf{B}_{\mathbf{c}}{ }^{T} \mathbf{K}_{\mathbf{c}} \mathbf{x}\right\|\right)^{T}, \\
& \mathbf{q}:=\left(0,2 \nu_{r}\left\|\mathbf{K}_{\mathbf{r}}\right\|, 2 \delta_{c}\right)^{T},
\end{aligned}
$$

and after some manipulations, we arrive at

$$
\frac{d}{d t} V(\mathbf{x}, \mathbf{y}) \leq-<\mathbf{z}, \mathbf{M z}>+<\mathbf{q}, \mathbf{z}>
$$

where

$$
\mathbf{M}:=\left(\begin{array}{ccc}
m_{1} & -m_{2} & -\alpha_{c}^{c} \\
-m_{2} & m_{3} & -\alpha_{c}^{r} \\
-\alpha_{c}^{c} & -\alpha_{c}^{r} & 2 \gamma
\end{array}\right)
$$

with $m_{1}:=\lambda_{\min }\left(\mathbf{Q}_{\mathbf{c}}\right), m_{2}:=\mu_{r}^{c}|| \mathbf{K}_{\mathbf{r}}||$ and $m_{3}:=\lambda_{\min }\left(\mathbf{Q}_{\mathbf{r}}\right)-$ $2 \mu_{r}^{r}\left\|\mathbf{K}_{\mathbf{r}}\right\|$. According to the Theorem of Hurwitz, we have that $\mathbf{M}$ is positive definite if and only if (i) $m_{1}>0$, (ii) $m_{1} m_{3}-$ $m_{2}^{2}>0$, (iii) $\operatorname{det}(\mathbf{M})>0$.

Condition (i) is satisfied by construction, since $\mathbf{Q}_{\mathbf{c}}$ is positive definite. Condition (ii) leads to

$$
\lambda_{\min }\left(\mathbf{Q}_{\mathbf{c}}\right)\left[\lambda_{\min }\left(\mathbf{Q}_{\mathbf{r}}\right)-2 \mu_{r}^{r}\left\|\mathbf{K}_{\mathbf{r}}\right\|\right]-\left(\mu_{r}^{c}\right)^{2}\left\|\mathbf{K}_{\mathbf{r}}\right\|^{2}>0
$$

This condition holds if and only if the following inequalities are satisfied:

$$
\mu_{r}^{r}<\frac{\lambda_{\min }\left(\mathbf{Q}_{\mathbf{r}}\right)}{2\left\|\mathbf{K}_{\mathbf{r}}\right\|}
$$

and

$$
\mu_{r}^{c}<\frac{\left(\lambda_{\min }\left(\mathbf{Q}_{\mathbf{c}}\right)\left[\lambda_{\min }\left(\mathbf{Q}_{\mathbf{r}}\right)-2 \mu_{r}^{r}|| \mathbf{K}_{\mathbf{r}} \|\right]\right)^{1 / 2}}{\left\|\mathbf{K}_{\mathbf{r}}\right\|} .
$$

Condition (iii) leads to

$$
\gamma>\frac{2 \alpha_{c}^{r} \alpha_{c}^{c} m_{2}+\left(\alpha_{c}^{c}\right)^{2} m_{3}+\left(\alpha_{c}^{r}\right)^{2} m_{1}}{2\left(m_{1} m_{3}-m_{2}^{2}\right)}
$$

Choosing $\mathbf{Q}_{\mathbf{c}}, \mathbf{Q}_{\mathbf{r}}$ and $\gamma$ according to (6)-(8), then $\mathbf{M}$ is positive definite. Now, we have

$$
\frac{d}{d t} V(\mathbf{x}, \mathbf{y}) \leq-\lambda_{\min }(\mathbf{M})\|\mathbf{z}\|^{2}+\|\mathbf{q}\|\|\mathbf{z}\| .
$$

Therefore, setting $r_{1}:=\frac{\lambda_{\min }(\mathbf{M})}{\|\mathbf{q}\|}$,

$$
\Gamma:=\left\{(\mathbf{x}, \mathbf{y}) \in \mathbb{R}^{n_{c}} \times \mathbb{R}^{n_{r}} \quad \text { such that } \mid \mathbf{z}(\mathbf{x}, \mathbf{y}) \|=r_{1}\right\}
$$


and $r:=\max _{(\mathbf{x}, \mathbf{y}) \in \Gamma}\|(\mathbf{x}, \mathbf{y})\|$, which is well-defined since $\Gamma$ is a compact set, we have that

$$
\frac{d}{d t} V(\mathbf{x}, \mathbf{y})<0
$$

holds for all $\|(\mathbf{x}, \mathbf{y})\|>r$.

The smallest level surface of $V(\mathbf{x}, \mathbf{y})$ containing $B_{r}$ (the ball centered at the origin of radius $r)$ is given by $E_{r}:=\{(\mathbf{x}, \mathbf{y}) \in$ $\mathbb{R}^{n_{c}} \times \mathbb{R}^{n_{r}}$, such that $\left.V(x, y)=\lambda_{\min }(\mathbf{K}) r^{2}=V_{r}\right\}$. If we denote $\rho$ the length of the major semi-axis of the hyper-ellipsoid $E_{r}$, then

$$
V(x, y)_{\mid E_{r}}=\lambda_{\min }(\mathbf{K}) r^{2}=\lambda_{\max }(\mathbf{K}) \rho^{2},
$$

which leads to

$$
\rho:=r \sqrt{\frac{\lambda_{\max }(\mathbf{K})}{\lambda_{\min }(\mathbf{K})}} .
$$

Therefore any trajectory $\mathbf{v}(t)=(\mathbf{x}(t), \mathbf{y}(t))$ enters the ball of ultimate boundedness $\mathrm{B}_{\rho}$, at a time $t=t_{*}$. Furthermore, as it is proved in [6] with all the details, the compact set $\mathcal{K}:=$ $\left\{(\mathbf{x}, \mathbf{y}) \in \mathbb{R}^{n_{c}} \times \mathbb{R}^{n_{r}}\right.$ such that $\left.V(\mathbf{x}, \mathbf{y}) \leq V_{r}\right\}=E_{r} \cup \operatorname{Int}\left(E_{r}\right)$ is a global uniform attractor for system (1).

Observe that $\alpha_{c}^{c}, \alpha_{c}^{r}, \delta_{c}, \mu_{r}^{c}, \mu_{r}^{r}$, and $\nu_{r}$ are bounds on the uncertainties, which are given a priori, while $\mathbf{Q}_{\mathbf{c}}, \mathbf{Q}_{\mathbf{r}}$ and $\gamma$ are free design parameters. According to the above stability analysis, the designer has to choose $\mathbf{Q}_{\mathbf{c}}, \mathbf{Q}_{\mathbf{r}}$ and $\gamma$ satisfying inequalities (6)-(8). These inequalities give only sufficient stability conditions, which in practice can be relaxed as it will be illustrated in the example in the next section.

\section{Bridge platform with moving vehicles}

In [5] a semi-active control approach for an elastically suspended bridge with crossing vehicles is provided. Figure $1 \mathrm{de}-$ picts the main elements of the simplified dynamic model. We will apply the active control approach described in Section 1 to that model. The bridge section consists of a rigid platform with elastic mounts on the left-hand and right-hand sides. The main variables that we will measure are the vertical deviation $z$ of the center of mass $C$ of the bridge and the inclination $\Theta$ with respect to the horizon of the platform. Within a time interval $\left[t_{0}, t_{f}\right]$, an object, say a truck, crosses the bridge. The truck is modelled by a mass $m$ with an elastic suspension (damping $c$ and stiffness $k$ ). The truck crosses the bridge with velocity $v(t)$. Additional variables $\xi, \eta$ and $\zeta$ are chosen according to Figure 1. The mass of the platform is given by $M$, and the moment of inertia with respect to $C$ by the parameter $J$.

The active control is implemented by two actuators located between the ground and the bridge at the left and the right ends respectively. The actuators $A_{1}$ and $A_{2}$ supply vertical control forces $M u_{1}$ and $M u_{2}$ which complement the resistant (passive) forces $F_{1}$ and $F_{2}$ given by the elastic supports. $u_{1}$ and $u_{2}$ are the control variables. The objective is that the active forces $M u_{1}$ and $M u_{2}$ significantly improve the attenuation of the vibration of the bridge caused by the crossing vehicle.

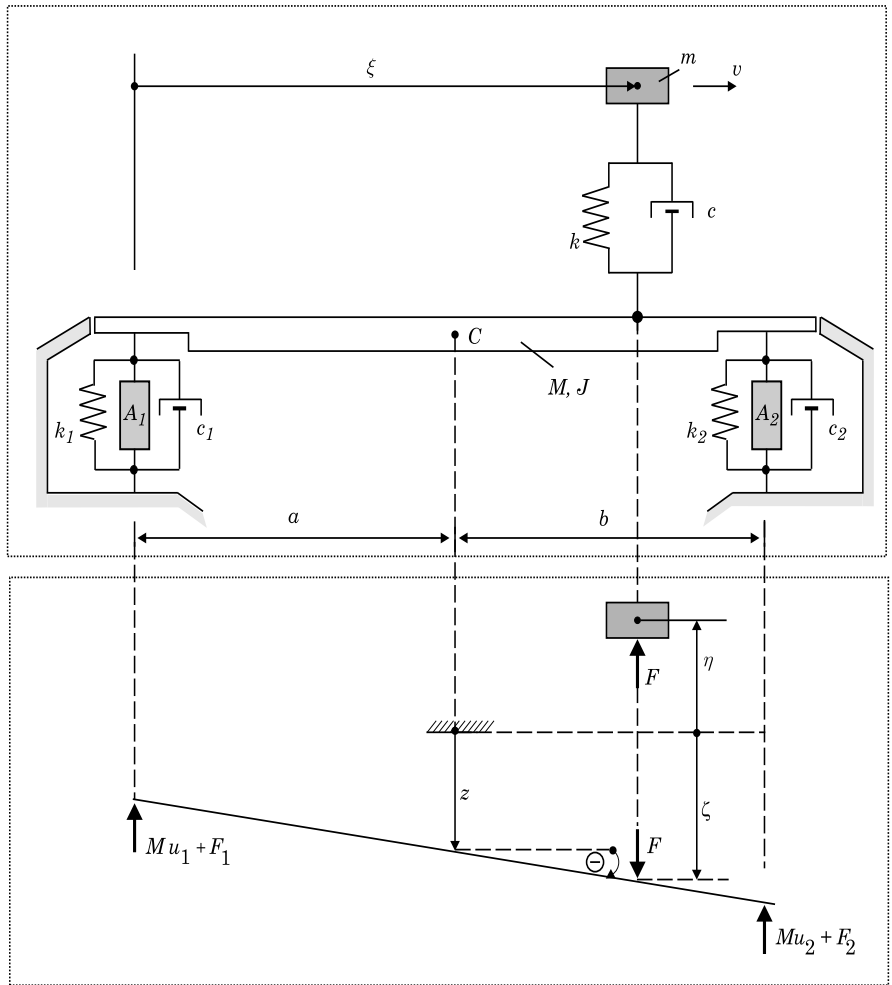

Figure 1: Model of an actively mounted platform bridge with one crossing vehicle.

\subsection{Equations of motion}

Without loose of generality we set $t_{0}:=0$. In the following $t_{f}$ denotes the final time of interaction between the structure and the truck. This value can be determined by physical sensors, hence it is not an uncertain value.

Equations of motion of the truck: For $t<0$ and $t>t_{f}$ the equation of motion of the truck is $m \ddot{\eta}=k \eta_{0}-m g$, where $\eta_{0}$ is the position of relaxed suspension. For $t \in\left[0, t_{f}\right]$ we have

$$
m \ddot{\eta}=F-m g,
$$

where $F:=k\left(\eta_{0}-(\eta+\zeta)\right)-c(\dot{\eta}+\dot{\zeta})$, and $\zeta:=z+(\xi-a) \Theta$, assuming small values of $\Theta$.

Equations of motion of the bridge: For $t<0$ the bridge is in a steady state. For $t \in\left[0, t_{f}\right]$ we have the following equations of motion:

$$
\begin{aligned}
& M \ddot{z}=M g+F-F_{1}-F_{2}-M u_{1}-M u_{2}, \\
& J \ddot{\Theta}=(\xi-a) F+a F_{1}-b F_{2}+a M u_{1}-b M u_{2} .
\end{aligned}
$$

where $F_{1}=k_{1}\left(-z_{1,0}+z-a \Theta\right)+c_{1}(\dot{z}-a \dot{\Theta})$, and $F_{2}=$ $k_{2}\left(-z_{2,0}+z+b \Theta\right)+c_{2}(\dot{z}+b \dot{\Theta})$, being $z_{1,0}$ (respectively $\left.z_{2,0}\right)$ the vertical position of relaxed left-hand (respectively righthand) suspension.

In order to use the model framework in (1), we consider the bridge as the main system and the truck as the attached system. The space state variables are split into the ones we measure, 
$\mathbf{x}:=(z, \Theta, \dot{z}, \dot{\Theta})^{T}$, and the ones we do not measure, $\mathbf{y}:=$ $(\eta, \dot{\eta})^{T}$. We also consider $\mathbf{u}:=\left(u_{1}, u_{2}\right)^{T}$. The interaction variable $\mathbf{w}=\mathbf{f}(\mathbf{x}, \mathbf{y}, t)$ is now due to the force $F$ between the bridge and the truck, that is the scalar function

$$
w=F=k\left(\eta_{0}-(\eta+\zeta)\right)-c(\dot{\eta}+\dot{\zeta})
$$

For $w=0$ the two systems are obviously decoupled. This occurs for $t>t_{f}$, and then the equations of motion of the bridge are

$$
\begin{aligned}
& M \ddot{z}=M g-F_{1}-F_{2}-M u_{1}-M u_{2}, \\
& J \ddot{\Theta}=a F_{1}-b F_{2}+a M u_{1}-b M u_{2} .
\end{aligned}
$$

We have to build the matrices $\mathbf{A}_{\mathbf{c}}, \mathbf{B}_{\mathbf{c}}$, and $\mathbf{A}_{\mathbf{r}}$ with known parameters, and the functions $\mathbf{g}_{\mathbf{c}}$ and $\mathbf{g}_{\mathbf{r}}$ including the coupling effects and the uncertainties.

In this model we consider that the structural parameters of the bridge $\left(M, J, c_{1}, c_{2}, k_{1}, k_{2}\right)$ are known, while the parameters related to the truck $\left(m, c, k, \eta_{0}, \xi, v\right)$ are uncertain. For these parameters, we assume that $\frac{k}{m}=\omega_{0}+\Delta \omega$, with $|\Delta \omega| \leq \bar{\omega}$, $\frac{c}{m}=\sigma_{0}+\Delta \sigma$, with $|\Delta \sigma| \leq \bar{\sigma}, \frac{k}{M}=\Omega$, with $\Omega \leq \bar{\Omega}, \frac{c}{M}=\Upsilon$, with $\Upsilon \leq \bar{\Upsilon},\left|\eta_{0}\right| \leq \bar{\eta}_{0},|\bar{v}(t)| \leq \bar{v}$. In this description $\omega_{0}$ and $\sigma_{0}$ are known nominal values and $\bar{\omega}, \bar{\sigma}, \bar{\Omega}, \bar{\Upsilon}, \bar{\eta}_{0}$ and $\bar{v}$ are known bounds.

Finally the equations of motion (10) and (11) can be re-written in the following state form:

$$
\begin{aligned}
& \dot{\mathbf{x}}=\mathbf{A}_{\mathbf{c}} \mathbf{x}+\mathbf{B}_{\mathbf{c}} \mathbf{u}+\mathbf{g}_{\mathbf{c}}(\mathbf{x}, \mathbf{w}, t)=\mathbf{A}_{\mathbf{c}} \mathbf{x}+\mathbf{B}_{\mathbf{c}} \mathbf{u}+\tilde{\mathbf{g}}_{\mathbf{c}}(\mathbf{x}, \mathbf{y}, t) \\
& \dot{\mathbf{y}}=\mathbf{A}_{\mathbf{r}} \mathbf{y}+\mathbf{g}_{\mathbf{r}}(\mathbf{y}, \mathbf{w}, t)=\mathbf{A}_{\mathbf{r}} \mathbf{y}+\tilde{\mathbf{g}}_{\mathbf{r}}(\mathbf{x}, \mathbf{y}, t)
\end{aligned}
$$

where

$$
\begin{gathered}
\mathbf{A}_{\mathbf{c}}=\left(\begin{array}{cccc}
0 & 0 & 1 & 0 \\
0 & 0 & 0 & 1 \\
-\frac{k_{1}+k_{2}}{M} & \frac{a k_{1}-b k_{2}}{M} & -\frac{c_{1}+c_{2}}{M} & \frac{a c_{1}-b c_{2}}{M} \\
\frac{a k_{1}-b k_{2}}{J} & -\frac{a^{2} k_{1}+b^{2} k_{2}}{J} & \frac{a c_{1}-b c_{2}}{J} & -\frac{a^{2} c_{1}+b^{2} c_{2}}{J}
\end{array}\right), \\
\mathbf{B}_{\mathbf{c}}=\left(\begin{array}{cc}
0 & 0 \\
0 & 0 \\
-1 & -1 \\
\frac{a M}{J} & -\frac{b M}{J}
\end{array}\right), \quad \text { and } \quad \tilde{\mathbf{g}}_{\mathbf{c}}=\left(\begin{array}{c}
0 \\
0 \\
g_{c, 3} \\
g_{c, 4}
\end{array}\right) .
\end{gathered}
$$

Here for $t \in\left[0, t_{f}\right]$ :

$$
\begin{aligned}
& g_{c, 3}(\mathbf{x}, \mathbf{y}, t):=-\frac{k}{M} z-\frac{1}{M}[k(\xi(t)-a)+c v] \Theta \\
& -\frac{c}{M} \dot{z}-\frac{c}{M}(\xi(t)-a) \dot{\Theta}-\frac{k}{M} \eta-\frac{c}{M} \dot{\eta} \\
& +\frac{k}{M} \eta_{0}+\frac{k_{1}}{M} z_{1,0}+\frac{k_{2}}{M} z_{2,0}+g
\end{aligned}
$$

and

$$
\begin{aligned}
& g_{c, 4}(\mathbf{x}, \mathbf{y}, t):=-\frac{k}{J}(\xi(t)-a) z-\frac{1}{J}\left[k(\xi(t)-a)^{2}\right. \\
& +c v(\xi(t)-a)] \Theta-\frac{c}{J}(\xi(t)-a) \dot{z}-\frac{c}{J}(\xi(t)-a)^{2} \dot{\Theta} \\
& -\frac{k}{J}(\xi(t)-a) \eta-\frac{c}{J}(\xi(t)-a) \dot{\eta}+\frac{k}{J}(\xi(t)-a) \eta_{0} \\
& -\frac{a k_{1}}{J} z_{1,0}+\frac{b k_{2}}{J} z_{2,0}
\end{aligned}
$$

while, for $t>t_{f}$, we have

$$
g_{c, 3}:=\frac{k_{1}}{M} z_{1,0}+\frac{k_{2}}{M} z_{2,0}+g
$$

and

$$
g_{c, 4}:=-\frac{a k_{1}}{J} z_{1,0}+\frac{b k_{2}}{J} z_{2,0}
$$

We also have

$$
\mathbf{A}_{\mathbf{r}}=\left(\begin{array}{cc}
0 & 1 \\
-\omega_{0} & -\sigma_{0}
\end{array}\right)
$$

and, for $t \in\left[0, t_{f}\right]$,

$$
\tilde{\mathbf{g}}_{\mathbf{r}}(\mathbf{x}, \mathbf{y}, t)=\left(\begin{array}{c}
0 \\
g_{r, 2}
\end{array}\right)
$$

where

$$
\begin{gathered}
g_{r, 2}=\frac{k}{m} z-\frac{1}{m}[k(\xi(t)-a)+c v] \Theta-\frac{c}{m} \dot{z} \\
-\frac{c}{m}(\xi(t)-a) \dot{\Theta}-\Delta \omega \eta-\Delta \sigma \dot{\eta}+\frac{k}{m} \eta_{0}-g
\end{gathered}
$$

For $t>t_{f}$, it is $\tilde{\mathbf{g}}_{\mathbf{r}}:=\left(0,-\Delta \omega \eta-\Delta \sigma \dot{\eta}+\frac{k}{m} \eta_{0}-g\right)^{T}$.

In [7] it is checked that the model described above satisfies the Assumptions 1 and 2 required in Section 2. This is necessary in order to ensure that the stability analysis developed in Section 3 holds.

\subsection{Tuning of the control law}

For the design of the control law, we use the following set of known parameters:

Bridge: $M=10^{5}[\mathrm{~kg}], J=2 \cdot 10^{7}\left[\mathrm{~kg} \mathrm{~m}^{2}\right], a=b=25$ $[\mathrm{m}], k_{i}=4 \cdot 10^{6}[\mathrm{~N} / \mathrm{m}]$ and $c_{i}=4 \cdot 10^{4}[\mathrm{Ns} / \mathrm{m}]$ for each $i=1,2$. $z_{1,0}=z_{2,0}=-0.125[\mathrm{~m}]$, which correspond to the equilibrium position for the platform without truck and no control.

Nominal parameters and bounds for uncertainties: $\bar{\eta}_{0}=1[\mathrm{~m}]$, $\omega_{0}=40[\mathrm{~N} /(\mathrm{m} \mathrm{kg})], \bar{\omega}=20[\mathrm{~N} /(\mathrm{m} \mathrm{kg})], \sigma_{0}=1[\mathrm{Ns} /(\mathrm{m} \mathrm{Kg})]$, $\bar{\sigma}=5[\mathrm{Ns} /(\mathrm{m} \mathrm{Kg})], \bar{\Omega}=5[\mathrm{~N} /(\mathrm{m} \mathrm{kg})], \bar{\Upsilon}=0.5[\mathrm{Ns} /(\mathrm{m} \mathrm{Kg})]$, $\bar{v}=8.33[\mathrm{~m} / \mathrm{s}](\bar{v}=30[\mathrm{~km} / \mathrm{h}]), k_{0}=4 \cdot 10^{5}[\mathrm{~N} / \mathrm{m}], c_{0}=10^{4}$ $[\mathrm{Ns} / \mathrm{m}]$.

We select $\mathbf{Q}_{\mathbf{r}}=\mathbf{I}$ (identity matrix) and

$$
\mathbf{Q}_{\mathbf{c}}=\left(\begin{array}{cccc}
5 & 0 & 0 & 0 \\
0 & 1 & 0 & 0 \\
0 & 0 & 1 & 0 \\
0 & 0 & 0 & 1
\end{array}\right)
$$

which gives

$$
-\mathbf{B}_{\mathbf{c}}{ }^{T} \mathbf{K}_{\mathbf{c}}=\left(\begin{array}{rrrr}
0.0312 & -0.0002 & 0.6641 & -0.0251 \\
0.0312 & 0.0002 & 0.6641 & 0.0251
\end{array}\right),
$$

and the gain matrix is given by $\mathbf{G}:=-\gamma \mathbf{B}_{\mathbf{c}}{ }^{T} \mathbf{K}_{\mathbf{c}}$. 
With the above selection of $\mathbf{Q}_{\mathbf{r}}$, the inequality (6) leads to

$$
\mu_{r}^{r}<\frac{\lambda_{\min }\left(\mathbf{Q}_{\mathbf{r}}\right)}{2\left\|\mathbf{K}_{\mathbf{r}}\right\|} \approx 0.024
$$

Taking $\mu_{r}^{r}=0.02$ and using the selected matrix $\mathbf{Q}_{\mathbf{c}}$, inequality (7) leads to

$$
\mu_{r}^{c}<\frac{\left(\lambda_{\min }\left(\mathbf{Q}_{\mathbf{c}}\right)\left[\lambda_{\min }\left(\mathbf{Q}_{\mathbf{r}}\right)-2 \mu_{r}^{r}\left\|\mathbf{K}_{\mathbf{r}}\right\|\right]\right)^{1 / 2}}{\left\|\mathbf{K}_{\mathbf{r}}\right\|} \approx 0.021 .
$$

These two inequalities impose maximum theoretical allowable values for the parameters $\mu_{r}^{r}$ and $\mu_{r}^{c}$ appearing in the affine bounding of the coupling uncertain function $\mathbf{g}_{\mathbf{r}}$ in Assumption 2.3.

In this case, taking $\mu_{r}^{r}=0.02$ and $\mu_{r}^{c}=0.02$, inequality (8) becomes

$$
\gamma \geq 3.2051 \cdot 10^{5}=: \gamma_{*}
$$

Inequalities (14), (15) and (16) give sufficient conditions to ensure that the control law satisfies the stability analysis of Section 3 . When computing the uncertainty bounds for the data set in this example, we obtain $\mu_{r}^{r} \approx 20.62$ and $\mu_{r}^{c} \approx 1558.4$, which are significantly bigger than the maximum allowable values according to the theoretical stability analysis. In practice, simulation results show that the control law guarantees the derived stability property even for these actual values. This is because the Lyapunov approach used in the stability analysis is rather conservative in giving sufficient stability conditions, which are based on rough estimates for the values of $\alpha_{c}^{c}, \alpha_{c}^{r}, \delta_{c}, \mu_{r}^{c}, \mu_{r}^{r}$, and $\nu_{r}$. Practice also shows that values of $\gamma$ lower than $\gamma_{*}$ are acceptable to achieve stability and satisfactory control results as it is illustrated next.

\subsection{Numerical example}

The following unknown parameters for the truck (which lie within the prescribed uncertainty bounds) are used:

Truck: $m=10^{4}[\mathrm{~kg}], v=8.33[\mathrm{~m} / \mathrm{s}](v=30[\mathrm{~km} / \mathrm{h}]), k=$ $4 \cdot 10^{5}[\mathrm{~N} / \mathrm{m}], c=10^{4}[\mathrm{Ns} / \mathrm{m}], \eta_{0}=0.75[\mathrm{~m}]$.

Figure 2 shows the time history of the vertical deflection $z$ of the center of mass $C$ of the platform for the uncontrolled case (dash line) and the controlled case (solid line) when using $\gamma=$ 20 . The platform is excited by the crossing of the truck for time $t \in[0,6]$ seconds, and after $t=6$ seconds the platform evolves with no excitation. In both the uncontrolled and the controlled cases, the center of masses evolves towards its new equilibrium position $(z=0.125[m])$ with the truck on the platform, and evolves to recover the initial equilibrium position after the truck leaves the platform at $t=6$ seconds. A significant reduction in the oscillations around the equilibrium positions of $C$ can be observed with the control operation.

Figure 3 shows that the inclination $\Theta$ of the bridge has a similar pattern for the uncontrolled and the controlled case. It behaves almost linearly while the truck crosses with a constant speed.

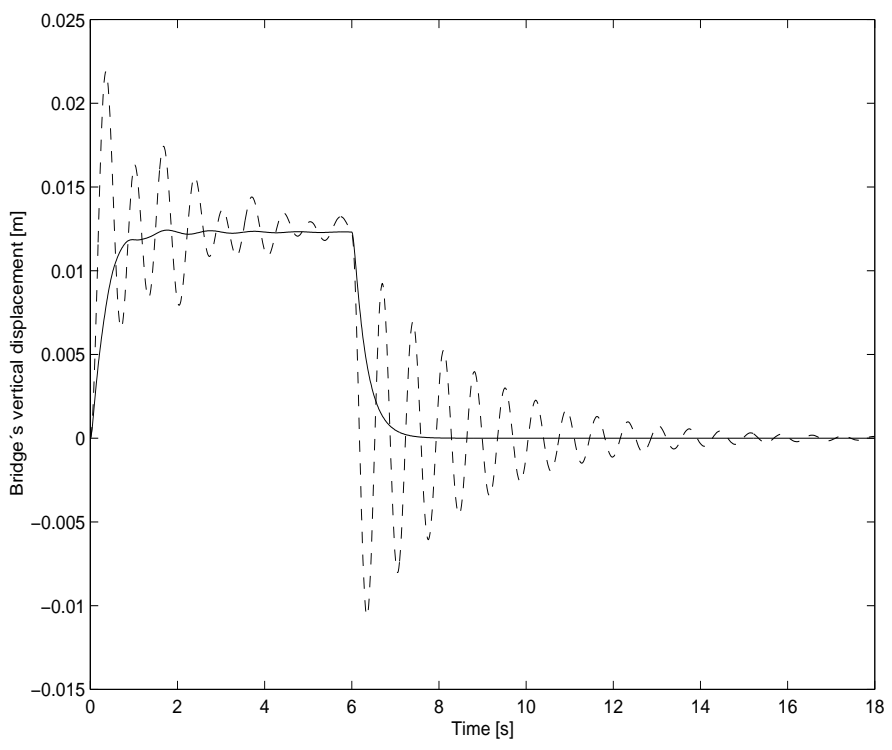

Figure 2: Time history of $z(t)$ without control (dash line) and with control (solid line) using $\gamma=20$.

After the truck leaves the bridge, the inclination tends to zero in a damped oscillatory motion.

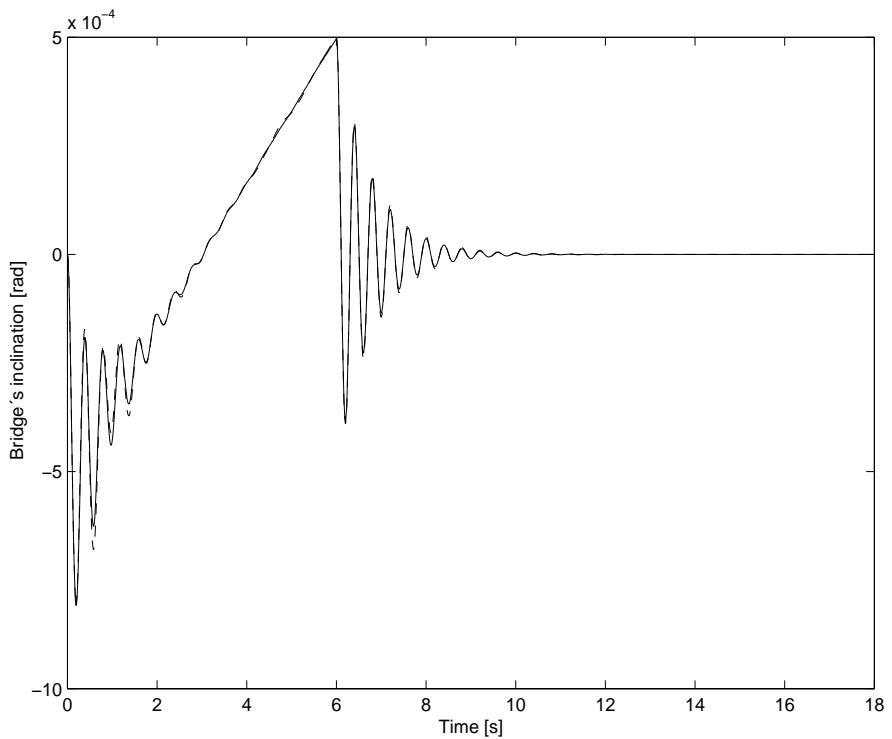

Figure 3: Time history of $\Theta(t)$ without control (dash line) and with control (solid line) using $\gamma=20$.

Figure 4 displays the control signals $u_{1}$ and $u_{2}$. Initial impulsive actions are observed when the excitation starts up and when it disappears, followed by smooth evolutions to zero.

\section{Conclusions}

A linear feedback control law is proposed to actively reduce the vibrations of a class of structures excited by the coupling with an uncertain system. The main practical advantage is the simplicity of the control law. It is composed by a nominal gain 

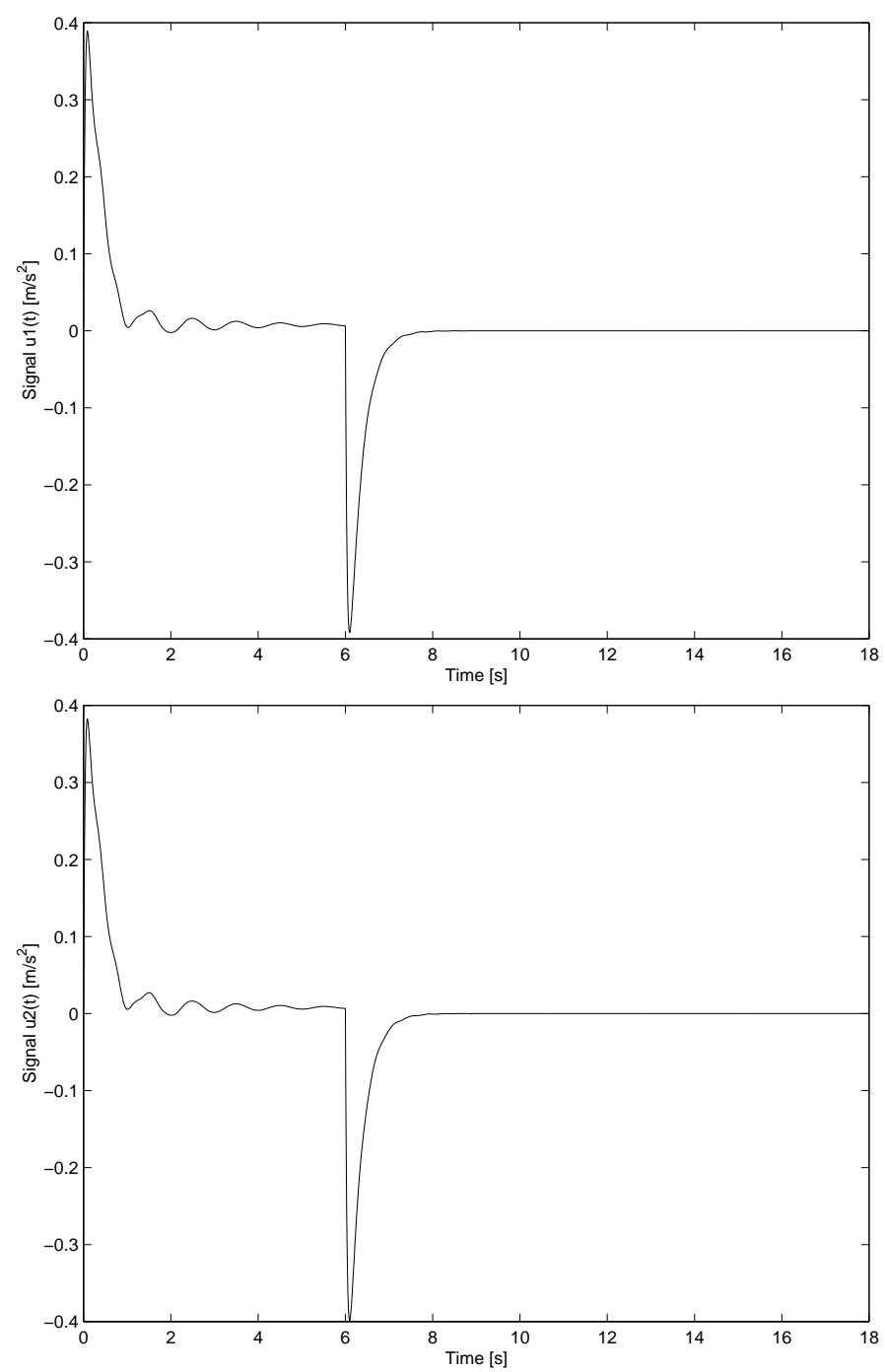

Figure 4: Time history of controls $u_{1}(t)$ and $u_{2}(t)$ when using $\gamma=$ 20 .

matrix with a scalar gain factor. The design of this matrix uses information only on the known linear part of the model of the main uncoupled structure. The selection of the scalar gain parameter is chosen using information on the known linear part of the model of the exciting coupled system, as well as on the bounds of two uncertain functions which describe the interaction between the controlled structure and the exciting system. The real time implementation requires feedback only of the structure state.

A Lyapunov stability analysis gives sufficient conditions for the existence of a ball of ultimate boundedness and a global uniform attractor for both the structure and the exciting system. Due to the character of the Lyapunov techniques, these conditions can appear to be conservative. In practice, such sufficient conditions can be relaxed keeping satisfactory performance in the control operation, as it is shown in the example presented in the paper, in which a model of a bridge platform is controlled against the excitation induced by a crossing vehicle.

\section{Acknowledgements}

This work has been partially supported by a Germany-Spain Acción Integrada HA1999-0095 and is part of a project funded by CICYT (TAP99-1079-C03) The second author also appreciates the support of a UPC grant PR99-08.

\section{References}

[1] G. Leitmann. "Guaranteed asymptotic stability for some linear systems with bounded uncertainties", Transactions of ASME 101, pp. 212-216, (1979).

[2] M. Corless, G. Leitmann. "Continuous state feedback guaranteeing uniform ultimate boundedness for uncertain dynamic systems", IEEE Transactions on Automatic Control, 26, pp. 1139-1144, (1987).

[3] J. Rodellar. "On a stability-based approach for robust active, semiactive and hybrid structural control", Structural Control for Civil and Infrastructure Engineering, F. Casciati, G. Magonette (eds.), pp. 487-494, World Scientific, (2001).

[4] G. Leitmann, E. Reithmeier. "Semiactive control of a vibrating system by means of electrorheological fluids", Dynamics and Control, 3, pp. 7-33, (1993).

[5] E. Reithmeier, G. Leitmann. "Structural vibration control", Journal of the Franklin Institute (2001). 338, pp. 203-223, (2001).

[6] J. Rodellar, G. Leitmann, E.P. Ryan. "Output feedback control of uncertain coupled systems", International Journal of Control, 58, pp. 445-457, (1993).

[7] J. Rodellar, V. Mañosa, E. Reithmeier, G. Ehret. “Active vibration control of a class of structures with uncertain couplings", Preprint Dept. Matemàtica Aplicada III (UPC) and IMR Uni-Hannover, (2001).

[8] A.H. Barbat, J. Rodellar, E.P. Ryan, N. Molinares. “Active control of non-linear base-isolated buildings", Journal of Engineering Mechanics ASCE, 121, pp. 676-684, (1993). 